\title{
A utilização de informantes-chave da comunidade na identificação de pessoas portadoras de alterações cinético-funcionais da cidade de Caxias do Sul, RS
}

\author{
The use of key informants from within the community \\ for identifying people with functional kinetic alterations \\ in Caxias do Sul, Rio Grande do Sul State, Brazil
}

Alexandra Renosto ${ }^{1}$

Jorge Luiz de AndradeTrindade 2

${ }^{1}$ Faculdade da Serra Gaúcha. Rua Os Dezoito do Forte 2366. 95020-472 Caxias do Sul RS.

alexandra.renosto@fsg.br

${ }^{2}$ Centro Universitário

Feevale - PUCRS

\begin{abstract}
Purpose: Remaining functionally active within a specific social context seems to be one of the hardest challenges faced by our society. The purpose of this paper is to study the epidemiological profiles of people with functional kinetic alterations as noted by key informants living in Caxias do Sul, Rio Grande do Sul State, Brazil, considering social, economic and cultural characteristics, as well as family/individual profiles and healthcare aspects, linked to functional capacities. Methodology: This research project included an observational descriptive study. The population studied consisted of people suffering from functional kinetic alterations noted by from key informants, known as chapel-keepers. The tools used were: questionnaires addressed to the chapel-keepers, structured interviews and a Barthel Index rating for the functionally challenged. Findings: Only $21.9 \%$ of the population under study is being treated through physiotherapy. The use of key informants is a feasible alternative for helping the functional diagnosis of this population group. The characteristics of the population under study indicate the absence of physiotherapy and the need for effective programs addressing this problem.
\end{abstract}

Key words Key informants, Functional capacity, Epidemiological profile
Resumo Objetivo: M anter-se funcionalmente ativo em um determinado contexto social, parece ser um dos grandes desafios para a sociedade da atualidade. 0 objetivo deste trabalho foi estudar o perfil epidemiológico de portadores de alterações cinético-funcionais, a partir de informantes-chave da comunidade de Caxias do Sul (RS), considerando características socioeconômi cas, socioculturais, familiares/ individuais e cuidados com a saúde, vinculando-as com a capacidade funcional. Material e métodos: Esta pesquisa compreendeu um estudo observacional descritivo. A população em estudo foi constituída pelos portadores de alterações cinético-funcionais provenientes de indicações de informantes-chave da comunidade, conhecidos como zeladoras de capelinha. 0 s instrumentos aplicados foram: questionário às zeladoras de capelinha, entrevista estruturada e Índice de Barthel aos incapacitados funcionais. Resultados: A penas 21,9\% da população estudada que possui dependência funcional realiza tratamento fisioterapêutico. 0 uso de informantes-chave é uma alternativa viável no auxílio do diagnóstico funcional deste grupo populacional. As características da população em estudo supõem exclusão de atendimento fisioterapêtico e a necessidade de programas efetivos na abordagem do problema.

Palavras-chave Informantes-chave, Capacidade funcional, Perfil epidemiológico 


\section{Introdução}

A capacidade funcional é a capacidade de manter as habilidades físicas e mentais necessárias a uma vida independente e autônoma. Do ponto de vista da saúde pública, a capacidade funcional surgecomo um novo conceito de saúdemais adequado para instrumentalizar e operacionalizar a atenção à saúde. Ações preventivas, assistenciais e de reabilitação devem objetivar a melhoria da capacidade funcional ou, no mínimo, a sua manutenção e, sempreque possível, a recuperação desta capacidade, uma vez perdida pelo indivíduo. Trata-se, portanto, de um enfoque que transcende o simples diagnóstico e tratamento de doenças específicas ${ }^{1}$.

Para uma efetiva mudança na abordagem da assistência à saúde, priorizando os aspectos de promoção, prevenção e reabilitação, provém a participação comunitária na detecção de suas necessidades. Visando a identificação dos portadores de alterações cinético-funcionais em um grande espaço populacional, apresenta-se como auxílio na pesquisa o informante da comunidade. Pessoas conhecedoras da história, de seus processos sociais e capazes de identificar os moradores de sua região. Pessoas que pelo tempo de residência em uma determinada área, pela posição e pelas relações sociais que têm dentro da comunidade, possuem conhecimento detal hado de características relevantes da mesma².

Como informante para a pesquisa, escolheuse uma personagem que preenche os requisitos propostos acima e que até hoje mantém viva a tradição católica de realizar a peregrinação da I magem de Nossa Senhora às famílias. Ela é denominada popularmente de zeladora de capelinha. Possui total domínio na região a que pertence, devido à predominância da religião católica, proporcionando informações de populações definidas, tendo como características a simplicidade, a rapidez eo voluntariado. Asinformantes são fontes não onerosas à pesquisa, sendo o alicerce para o deslinde do trabalho.

Baseando-senessa ótica, foi realizado um estudo observacional descritivo, de paradigma quantitativo, com os portadores de alterações cinético-funcionais do município de Caxias do Sul, RS. 0 território envolvido na investigação limitou-se à zona urbana do município. 0 trabal ho teve como objetivo identificar o perfil epidemiológico de pessoas portadoras de alterações cinético-funcionais, a partir de informantes-chave da comunidade, considerando características socioeconômicas, socioculturais, cuidados com a saúde efamiliares/individuais, vinculando-as à capacidade funcional.

\section{Material e métodos}

0 território envolvido na investigação limitouse à zona urbana do município de Caxias do Sul, localizado na extremidade leste da encosta superior do nordeste do Estado do Rio Grande do Sul. A zona urbana do município é composta por quarenta bairros regularizados, totalizando uma população de 333.391 habitantes. ${ }^{3}$ Os instrumentos de coleta de dados utilizados nesta pesquisa compreenderam um questionário, uma entrevista estruturada e o I Indice de Barthel.

\section{População em estudo}

As zeladoras de capelinha totalizaram uma população de 2.235 pessoas, independente da idade, sexo, cor ou raça, sendo considerado como perda de parte da amostra a omissão do preenchimento ea não entrega do questionário na data predefinida. A população selecionada caracterizou-se por 1.455 zeladoras, sendo todas do sexo feminino e com idades variando entre 40 e 70 anos.

\section{Procedimentos}

Os procedimentos do estudo foram divididos em seis fases, as quais são apresentadas a seguir:

Primeira fase: após a constatação da eficácia do instrumento, através da realização do projeto piloto, iniciou-se os encontros para aplicação do questionário às zeladoras de capelinha. Em seguida, procedeu-se o manejo das informações coletadas pelas senhoras leigas. Os dados foram transportados para uma tabela que continha as informações para a detecção dos portadores de alteração funcional.

Segunda fase: compreendeu a divisão daárea de estudo epidemiológico a partir de mapa do município e a aprovação dos instrumentos de coleta de dados aos portadores de alterações cinético-funcionais. Esseinstrumento baseou-sena utilização de variáveisindependentes divididas em quatro blocos: socioeconômico, sociocultural, familiar/individual e saúde, conforme apresentado no Quadro I. 
Quadro 1. Variáveis independentes utilizadas na pesquisa com portadores de alterações cinético-funcionais do município de Caxias do Sul, RS, 2003.

\begin{tabular}{|ll|}
\hline Bloco socioeconômico: & Renda familiar, escolaridade, tipo de moradia. \\
\hline Bloco sociocultural: & Lazer, participação na comunidade, religião. \\
\hline Bloco familiar e individual: & $\begin{array}{l}\text { Número de pessoas na família, cuidador, estado } \\
\text { civil, idade, sexo. }\end{array}$ \\
\hline Bloco saúde: & $\begin{array}{l}\text { Tipo de assistência à saúde, atendimento } \\
\text { fisioterapêutico, tipo de assistência fisioterapêutica } \\
\text { e morbidade referida. }\end{array}$ \\
\hline
\end{tabular}

Para a devida avaliação do nível de incapacidade do portador de alteração cinético-funcional, foi aplicado o Índice de Barthel, que medeo grau de assistência exigido por um indivíduo em dez itens deAVDs, envolvendo mobilidade e cuidados pessoais. Os níveis de mensuração estão limitados à independência completa ou à necessidade de assistência. Cada item do desempenho é avaliado em uma escala ordinal, com um número específico de pontos assinalados para cada nível ou classificação. Pesos variáveis foram estabelecidos para cada item, baseado no julgamento clínico e em outros critérios implícitos. Os escores atribuídos variam entre 0 (dependente) , 5 (necessidade de aju da ou supervisão), 10 (parcialmente dependente) e 15 (independente), este último considerado somente na transferência cadeira/cama e deambulação. Ao todo, somamse 100 pontos, traduzindo o nível dedependência da pessoa (0-15 = dependência total; 20-35 = dependência grave; 40-55 = dependência moderada; $60-95$ = dependência leve; 100 = independente). Esta escala, desenvolvida para avaliação do nível de dependência, principalmente para pacientes idosos ou portadores de seqüelas de doenças crônicas, vem sendo utilizada desde 1965 e é recomendada pela O PS/OM S, tendo em vista a facilidade de aplicação e adaptação a diferentes culturas. No Brasil, em pesquisa bibliográfica realizada, observa-se sua utilização com a mesma finalidade e versão traduzida ${ }^{4,5}$.

Terceira fase: realizou-se a pesquisa de campo com os portadores dealterações cinético-funcionais. Ao final, foi realizada a entrada de dados no Programa Epi Info versão 6.0, com dupla entrada e posterior comparação para avaliar a con- sistência da di gitação. A análisefoi realizada através do Programa SPSS versão 11.0. Para associação das variáveis ao estudo, foi aplicado o Teste Qui-quadrado, considerando-se como valor estatisticamente significativo $p<=0,05$.

0 projeto desta pesquisa foi submetido e aprovado pelo Comitê de Ética e Pesquisa do Centro Universitário Feevale, RS. Na coleta de dados, os aspectos éticos foram preservados através do Termo de Consentimento Informado.

\section{Análisee discussão dos resultados}

Foram identificadas pelas zeladoras de capelinha 480 pessoas com provável incapacidade funcional. Dessas, 142 pessoas foram consideradas como perdas, dentre elas, 65 não foram localizadas, 22 recusaram-se a participar do estudo e 55 pessoas haviam falecido, restando 338 pessoas.

Entre as perdas, as recusas e os não encontrados mostraram-se insignificantes, tendo em vista a dimensão do território a ser investigado. Entretanto, o número de 55 óbitos, sendo estes pessoas com idade predominante acima dos 60 anos, foi significativamente alto, considerando 0 curto período de apenas três meses despendido entre a coleta de informações com as zeladoras de capelinha e a investigação domiciliar.

\section{Capacidade funcional}

O total depessoas não institucionalizadas que apresentaram alguma incapacidade para o desempenho de suas atividades de vida diária representou cerca de $89,3 \%$ da população estuda- 
$\mathrm{da}$, sendo agrupada em dependentes leves e moderados $(56,2 \%)$ e dependentes graves e totais $(33,1 \%)$. A freqüência do problema na região urbana de Caxias do Sul segundo a metodologia adotada foi de $0,1 \%$ da população total $(\mathrm{N}=333.391)$.

0 baixo índice (10,7\%) de entrevistados que obtiveram independência demonstrou que os informantes utilizados na identificação da amostra em estudo foram eficazes nas informações fornecidas à pesquisadora, tendo uma margem de erro relativamente baixa. Todavia, essas pessoas apresentam al guma doença ou alteração em sua saúde, com chances de progredir para uma dependência funcional. A pesar do número significativo de perdas das participantes, pode-secomprovar a dinâmica e a importância dessas senhoras na comunidade, no auxílio em estudos epidemiológicos.

\section{Características familiares/individuais e socioeconômicas}

$\mathrm{Na}$ Tabela 1 são apresentados os dados referentes às características socioeconômicas efamiliares/individuais 0 s dependentes funcionais representando $46,5 \%$ da população total encontram-se na faixa etária acima dos 60 anos, refletindo $o$ aumento da longevidade e a maior susceptibilidade aos agravos da saúde, comprometimento da funcionalidadee independência.

Estudos anteriores observam a predominância do sexo feminino na dependência funcional, 0 que foi confirmado no presente estudo. Enquanto as mulheres possuem a vantagem da longevidade, elas são vítimas mais freqüentes da violência doméstica e de discriminação no acesso à educação, salário, alimentação, trabal ho significativo, cuidados da saúde, heranças, medidas de seguro social e poder político. Essas desvantagens cumulativas significam que as mulheres, mais que os homens, têm maior inclinação para a pobreza e o sofrimento de deficiências $5^{6,7,1}$.

Quanto à condição de estado civil, as variáveis estudadas mostram-se dispersas, não demonstrando significância estatística. Entretanto, pode-se evidenciar que o estado civil solteiro, viúvo e desquitado repercute de forma negativa na capacidade funcional, em comparação com o estado casado ou com companheiro. Além disso, pode se notar que as pessoas dependentes funcionais não moram só e os que permanecem neste estado possuem uma condição melhor de autonomia em relação aos demais. Pessoas com incapacidade funcional apresentam maior vulnera- bilidade à solidão e isolamento social, sendo um fator negativo no processo de desenvolvimento da capacidade funcional ${ }^{6}$.

O cuidador é aquele que possui a total ou maior responsabilidade pelos cuidados prestados ao doente crônico em seu domicílio. Nesse estudo, destaca-se como cuidador o representante familiar, que exerce a atividade basi camente no espaço doméstico, facilitando a assistência necessária. Devido ao alto custo que os cuidados com uma pessoa incapacitada demanda, supõese que ocorra uma reorganização dos papéis familiares. 0 papel do cuidador, então, édesempenhado por um familiar, por tais motivos. No Brasil, os cuidadores são, em geral, familiares, sendo definidos como cuidadores informai $5^{8,9}$.

Em relação aos aspectos socioeconômicos, pode-se verificar que a maioria dos estudados possui um alto índice de baixa escolaridade e analfabetismo. Os baixos níveis de educação e analfabetismo estão associados a maiores riscos de deficiência e morte durante o processo desenvolvimento da vida ${ }^{10}$.

0 tipo de moradia demonstrou relevância estatística. A moradia segura e apropriada é essencial para o bem-estar do jovem e do idoso. Para esses, a localização, incluindo a proximidade de membros da família e serviços de transporte, podesignificar a diferença entre uma interação social positiva e o isolamento ${ }^{6}$.

É notável o baixo índice de renda familiar acima de 5 salários mínimos, demonstrando que esta variável está intimamente relacionada à saúde. As pessoas que possuem melhores condições financeiras possuem menor chance de apresentarem níveis de dependência funcional, por terem melhores condições detratamento e reabilitação ${ }^{11}$.

\section{Características socioculturais}

$\mathrm{Na}$ condição sociocultural, o grupo pesquisado com dependência funcional referiu alguma forma de ocupação do tempo livre. Porém restrito ao ambiente doméstico, como assistir televisão e ouvir rádio. Já na participação comunitária, o número de participantes é baixo e direcionado às pessoas com dependência leve ou moderada. Atribui-se o fato dos participantes não atuarem em atividades na comunidade provavelmenteà dificuldade de deslocamento.

Entretanto, atividades aparentemente corriqueiras, como freqüentar a igreja e participar de atividades religiosas como identificados na presente pesquisa, tornam-se muito importantes e parecem traduzir a idéia de vida ativa ${ }^{6}$. 
Tabela 1. Distribuição da população estudada segundo as características familiares/individuais e socioeconômicas. Caxias do Sul, 2003.

\begin{tabular}{|c|c|c|c|c|c|c|c|c|c|c|}
\hline \multirow{2}{*}{ Variáveis } & \multirow{2}{*}{ Categorias } & \multicolumn{2}{|c|}{ Independente } & \multicolumn{2}{|c|}{$\begin{array}{l}\text { Dependência leve } \\
\text { ou moderada }\end{array}$} & \multicolumn{2}{|c|}{$\begin{array}{l}\text { Dependência } \\
\text { grave ou total }\end{array}$} & \multicolumn{2}{|c|}{$\begin{array}{l}\text { População } \\
\text { em estudo }\end{array}$} & \multirow{2}{*}{$p$} \\
\hline & & $\mathbf{n}$ & $\%$ & $\mathbf{n}$ & $\%$ & $\mathbf{n}$ & $\%$ & $\mathbf{n}$ & $\%$ & \\
\hline \multirow{5}{*}{ Estado civil } & Solteiro & 15 & 4,4 & 64 & 18,9 & 54 & 16,0 & 133 & 39,3 & \\
\hline & Viúvo & 9 & 2,7 & 56 & 16,6 & 30 & 8,9 & 95 & 28,1 & \\
\hline & Casado/companheiro & 12 & 3,6 & 62 & 18,3 & 25 & 7,4 & 99 & 29,3 & \\
\hline & D esquitado/divorciado & 0 & 0,0 & 8 & 2,4 & 3 & 0,9 & 11 & 3,3 & 0,085 \\
\hline & Total & 36 & 10,7 & 190 & 56,2 & 112 & 33,1 & 338 & 100,0 & - \\
\hline \multirow{6}{*}{$\begin{array}{l}\text { Número de } \\
\text { pessoas que } \\
\text { moram na } \\
\text { residência }\end{array}$} & Uma & 5 & 1,5 & 12 & 3,6 & 1 & 0,3 & 18 & 5,3 & \\
\hline & Duas & 8 & 2,4 & 48 & 14,2 & 18 & 5,3 & 74 & 21,9 & \\
\hline & Três & 13 & 3,8 & 64 & 18,9 & 25 & 7,4 & 102 & 30,2 & \\
\hline & Quatro & 3 & 0,9 & 37 & 10,9 & 32 & 9,5 & 72 & 21,3 & \\
\hline & Cinco ou mais & 7 & 2,1 & 29 & 8,6 & 36 & 10,7 & 72 & 21,3 & $<0,001$ \\
\hline & Total & 36 & 10,7 & 190 & 56,2 & 112 & 33,1 & 338 & 100,0 & - \\
\hline \multirow{5}{*}{ Cuidador } & Familiar & 19 & 5,6 & 151 & 44,7 & 96 & 28,4 & 266 & 78,7 & \\
\hline & Pessoa contratada & 2 & 0,6 & 26 & 7,7 & 13 & 3,8 & 41 & 12,1 & \\
\hline & Ninguém & 13 & 3,8 & 12 & 3,6 & 2 & 0,6 & 27 & 8,0 & \\
\hline & Outro & 2 & 0,6 & 1 & 0,3 & 1 & 0,3 & 4 & 1,2 & $<0,001$ \\
\hline & Total & 36 & 10,7 & 190 & 56,2 & 112 & 33,1 & 338 & 100,0 & - \\
\hline \multirow{9}{*}{ Escolaridade } & Analfabeto & 6 & 1,8 & 50 & 14,8 & 53 & 15,7 & 109 & 32,2 & \\
\hline & Aprendeu fora da escola & 1 & 0,3 & 12 & 3,6 & 3 & 0,9 & 16 & 4,7 & \\
\hline & 1o grau incompleto & 22 & 6,5 & 102 & 30,2 & 45 & 13,3 & 169 & 50,0 & \\
\hline & 1o grau completo & 3 & 0,9 & 10 & 3,0 & 3 & 0,9 & 16 & 4,7 & \\
\hline & 20 grau incompleto & 2 & 0,6 & 2 & 0,6 & 1 & 0,3 & 5 & 1,5 & \\
\hline & 2 grau completo & 2 & 0,6 & 9 & 2,7 & 5 & 1,5 & 16 & 4,7 & \\
\hline & Superior incompleto & 0 & 0,0 & 2 & 0,6 & 1 & 0,3 & 3 & 0,9 & \\
\hline & Superior completo & 0 & 0,0 & 3 & 0,9 & 1 & 0,3 & 4 & 1,2 & 0,001 \\
\hline & Total & 36 & 10,7 & 190 & 56,2 & 112 & 33,1 & 338 & 100,0 & - \\
\hline \multirow{6}{*}{$\begin{array}{l}\text { Tipo } \\
\text { de moradia }\end{array}$} & Própria & 28 & 8,3 & 150 & 44,4 & 77 & 22,8 & 255 & 75,4 & \\
\hline & Alugada & 1 & 0,3 & 11 & 3,3 & 11 & 3,3 & 23 & 6,8 & \\
\hline & Cedida & 2 & 0,6 & 8 & 2,4 & 7 & 2,1 & 17 & 5,0 & \\
\hline & Familiar & 5 & 1,5 & 15 & 4,4 & 7 & 2,1 & 27 & 8,0 & \\
\hline & Invadida & 0 & 0,0 & 6 & 1,8 & 10 & 3,0 & 16 & 4,7 & 0,020 \\
\hline & Total & 36 & 10,7 & 190 & 56,2 & 112 & 33,1 & 338 & 100,0 & - \\
\hline \multirow{6}{*}{$\begin{array}{l}\text { Renda } \\
\text { familiar }\end{array}$} & $1 \mathrm{SM}$ & 9 & 2,7 & 38 & 11,2 & 17 & 5,0 & 64 & 18,9 & \\
\hline & $M$ aior que 1 SM até 2 SM & 8 & 2,4 & 48 & 14,2 & 28 & 8,3 & 84 & 24,9 & \\
\hline & $M$ aior que 2 SM até 3 SM & 9 & 2,7 & 45 & 13,3 & 30 & 8,9 & 84 & 24,9 & \\
\hline & $M$ aior que 3 SM até 5 SM & 7 & 2,1 & 35 & 10,4 & 24 & 7,1 & 66 & 19,5 & \\
\hline & Acima de 5 SM & 3 & 0,9 & 24 & 7,1 & 13 & 3,8 & 40 & 11,8 & $>0,100$ \\
\hline & Total & 36 & 10,7 & 190 & 56,2 & 112 & 33,1 & 338 & 100,0 & - \\
\hline
\end{tabular}

Fonte: Pesquisa de campo (2003).

A religião católica foi predominante, confirmando a atuação e distribuição das zeladoras de capelinha no município. Observou-se outras crenças relacionadas, mesmo tendo o predomínio da religião católica. Salienta-se que, durante o processo de aplicação do instrumento com as 
senhoras, essas foram instruídas para inclusão na pesquisa de todos os incapacitados funcionais, independente da religião praticada.

\section{Cuidados com a saúde}

A Tabela 2 apresenta a distribuição da população estudada em relação aos cuidados com a saúde. Somente $21,9 \%$ são dependentes funcionais que realizam acompanhamento fisioterapêutico. Número irrisório frente aos agravos que a degeneração funcional traz à saúde desses indivíduos. Desses, o número de indivíduos que utilizam o serviço de fisioterapia pelo Sistema Ú nico de Saúde érelativamente baixo.

$\mathrm{Na}$ identificação da morbidade referida ( Tabela 3) verificou-se o elevado número de pessoas dependentes com acometimento das doenças do aparelho circulatório, seguidas pelas doenças do sistema nervoso e doenças do sistema osteomuscular e do tecido conjuntivo. Os re- sultados comprovam que as doenças crônicas, em sua maioria, são as predominantes no grupo em discussão. Pesquisas demonstram que as origens do risco de doenças crônicas, como diabetes e doenças do coração, começam na infância ou na gestação. E este risco é subseqüentemente definido e modificado por fatores como status socioeconômico e experiências ao longo da vida ${ }^{10}$.

\section{Considerações finais}

As características apresentadas nesse trabalho estão atreladas umas às outras e requerem reflexões e intervenções primárias que possam influenciar positivamente no processo de degeneração funcional da população em estudo. Sugerese, então, o prolongamento de pesquisas sobre este estudo, objetivando a criação de programas e ações, com intuito de sanar esse problema.

Tabela 2. Distribuição da população estudada segundo os cuidados com a saúde. Caxias do Sul, 2003.

\begin{tabular}{|c|c|c|c|c|c|c|c|c|c|c|}
\hline \multirow[t]{2}{*}{ Variáveis } & \multirow[t]{2}{*}{ Categorias } & \multicolumn{2}{|c|}{ Independente } & \multicolumn{2}{|c|}{$\begin{array}{l}\text { Dependência leve } \\
\text { ou moderada }\end{array}$} & \multicolumn{2}{|c|}{$\begin{array}{l}\text { Dependência } \\
\text { grave ou total }\end{array}$} & \multicolumn{2}{|c|}{$\begin{array}{l}\text { População } \\
\text { em estudo }\end{array}$} & \multirow[t]{2}{*}{$p$} \\
\hline & & $\mathbf{n}$ & $\%$ & $n$ & $\%$ & $\mathbf{n}$ & $\%$ & $\mathbf{n}$ & $\%$ & \\
\hline \multirow{4}{*}{$\begin{array}{l}\text { Tipo de } \\
\text { assistência à } \\
\text { saúde }\end{array}$} & SUS & 10 & 3,0 & 78 & 23,1 & 54 & 16,0 & 142 & 42,0 & \\
\hline & Particular & 1 & 0,3 & 9 & 2,7 & 8 & 2,4 & 18 & 5,3 & \\
\hline & Plano de saúde & 25 & 7,4 & 103 & 30,5 & 50 & 14,8 & 178 & 52,7 & 0,060 \\
\hline & Total & 36 & 10,7 & 190 & 56,2 & 112 & 33,1 & 338 & 100,0 & 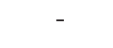 \\
\hline \multirow{3}{*}{$\begin{array}{l}\text { Realização de } \\
\text { atendimento } \\
\text { fisioterapêutico }\end{array}$} & Sim & 8 & 2,4 & 39 & 11,5 & 35 & 10,4 & 82 & 24,3 & \\
\hline & Não & 28 & 8,3 & 151 & 44,7 & 77 & 22,8 & 256 & 75,7 & 0,100 \\
\hline & Total & 36 & 10,7 & 190 & 56,2 & 112 & 33,1 & 338 & 100,0 & - \\
\hline \multirow{5}{*}{$\begin{array}{l}\text { Realização da } \\
\text { fisioterapia }\end{array}$} & SUS & 2 & 2,4 & 6 & 7,3 & 8 & 9,8 & 16 & 19,5 & \\
\hline & Plano de saúde & 3 & 3,7 & 17 & 20,7 & 10 & 12,2 & 30 & 36,6 & \\
\hline & Particular & 0 & 0,0 & 9 & 11,0 & 8 & 9,8 & 17 & 20,7 & \\
\hline & Outro & 3 & 3,7 & 8 & 9,8 & 8 & 9,8 & 19 & 23,2 & $>0,100$ \\
\hline & Total & 8 & 9,8 & 40 & 48,8 & 34 & 41,5 & 82 & 100,0 & - \\
\hline
\end{tabular}

Fonte: Pesquisa decampo (2003). 
Tabela 3. Distribuição da população estudada segundo a morbidade referida. Caxias do Sul, 2003.

\begin{tabular}{|c|c|c|c|c|c|c|c|c|c|}
\hline \multirow{2}{*}{ Categorias } & \multicolumn{2}{|c|}{ Independente } & \multicolumn{2}{|c|}{$\begin{array}{c}\text { Dependência leve } \\
\text { ou moderada }\end{array}$} & \multicolumn{2}{|c|}{$\begin{array}{l}\text { Dependência } \\
\text { grave ou total }\end{array}$} & \multicolumn{2}{|c|}{$\begin{array}{l}\text { População } \\
\text { em estudo }\end{array}$} & \multirow{2}{*}{$p$} \\
\hline & $\mathbf{n}$ & $\%$ & $\mathbf{n}$ & $\%$ & $\mathbf{n}$ & $\%$ & $\mathbf{n}$ & $\%$ & \\
\hline DIP & 0 & 0,0 & 0 & 0,0 & 1 & 0,3 & 1 & 0,3 & \\
\hline $\mathrm{N}$ & 1 & 0,3 & 2 & 0,6 & 0 & 0,0 & 3 & 0,9 & \\
\hline DS & 0 & 0,0 & 1 & 0,3 & 0 & 0,0 & 1 & 0,3 & \\
\hline DENM & 3 & 0,9 & 5 & 1,5 & 1 & 0,3 & 9 & 2,7 & \\
\hline TMC & 3 & 0,9 & 7 & 2,1 & 4 & 1,2 & 14 & 4,1 & \\
\hline DSN & 2 & 0,6 & 33 & 9,8 & 38 & 11,2 & 73 & 21,6 & \\
\hline DO & 1 & 0,3 & 2 & 0,6 & 1 & 0,3 & 4 & 1,2 & \\
\hline DOV & 1 & 0,3 & 0 & 0,0 & 0 & 0,0 & 1 & 0,3 & \\
\hline$D C$ & 8 & 2,4 & 59 & 17,5 & 29 & 8,6 & 96 & 28,4 & \\
\hline DR & 0 & 0,0 & 3 & 0,9 & 1 & 0,3 & 4 & 1,2 & \\
\hline $\mathrm{DAD}$ & 1 & 0,3 & 0 & 0,0 & 0 & 0,0 & 1 & 0,3 & \\
\hline DOTC & 13 & 3,8 & 49 & 14,5 & 8 & 2,4 & 70 & 20,7 & \\
\hline DAG & 0 & 0,0 & 1 & 0,3 & 2 & 0,6 & 3 & 0,9 & \\
\hline APN & 0 & 0,0 & 2 & 0,6 & 3 & 0,9 & 5 & 1,5 & \\
\hline$M C$ & 3 & 0,9 & 11 & 3,3 & 10 & 3,0 & 24 & 7,1 & \\
\hline CEM & 0 & 0,0 & 15 & 4,4 & 14 & 4,1 & 29 & 8,6 & $<0,001$ \\
\hline Total & 36 & 10,7 & 190 & 56,2 & 112 & 33,1 & 338 & 100,0 & - \\
\hline
\end{tabular}

Fonte: Pesquisa de campo (2003).

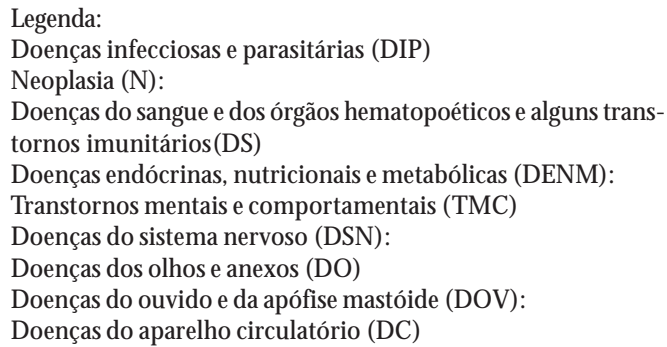

Doenças do aparelho respiratório (DR): Doenças do aparel ho digestivo (DAD)

Doenças de pelee detecido subcutâneo (DPSC):

Doenças do sistema osteomuscular e do tecido conjuntivo (DOTC)

Doenças do aparelho genito-urinário (DAG)

Algumas afecções originadas no período perinatal (APN)

$M$ alformações congênitas, deformidades e anomalias cromossômicas (MC)

Causas externas de morbidade (CEM)

\section{Colaboradores}

A Renosto participou da elaboração do marco teórico, estruturação do artigo, delineamento do método, elaboração do banco de dados e análise dos resultados. J Trindade participou da estruturação do artigo, delineamento do método, da orientação e da análise da discussão/resultados.

\section{Agradecimentos}

Aos acadêmicos de fisioterapia do Centro Universitário Feevale e Universidade de Caxias do Sul, que auxiliaram na coleta de dados e a Sra. Elisabeth $\mathrm{M}$ arcon, diretora da Elétrica M arcon e o Sr. Gilmar Isoton, Diretor da M aeli, patrocinadores do estudo. 


\section{Referências}

1. Veras RP. Terceira idade: gestão contemporânea em saúde. Rio de Janeiro: Relume; 2002.

2. OPS. Organização Pan-americana de Saúde. Escritório Regional da Organização Mundial da Saúde. A estimativa rápida e a divisão no distrito sanitário. $M$ anual de Instruções. Série Desenvolvimento de Serviços de Saúde n¹1. Brasília; 1993.

3. IBGE. Instituto Brasileiro de Geografia e Estatística. Censo Demográfico 2000. M alha M unicipal Digital do Brasil 1997. [acessado $2003 \mathrm{M}$ ar 01]. Disponível em: http://www.ibge.gov.br

4. Sullivan SBO, Schmittz TJ. Fisioterapia avaliação e tratamento. 2ae ed. São Paulo: Manole; 1993.

5. Cid-Ruzafa J, Damián M, Moreno J. Valoriación de la discapacidad fisica: el indice de Barthel. Revista Salud Publica 1997; 71(2).

6. Rosa TEC, et al. Fatores determinantes da capacidade funcional entre idosos. Revista Saúde Pública 2003.
7. Ramos LR. Fatores determinantes do envelhecimento saudável em idosos residentes em centro urbano: Projeto Epidoso, São Paulo. C Saúde Pública 2003; 19 (3): 793-798.

8. Guedes SL. A concepção sobre a família na geriatria e gerontologia brasileiras: ecos do dilema da multidisciplineridade. Revista Brasileira de Estudos Sociais 2000; 15(43).

9. Karsch UMS. Envelhecimento com dependência: revelando cuidadores. São Paulo: Educ; 1998.

10. OM S.Organização M undial de Saúde. Envelhecimento ativo: um projeto de política de saúde. Segundo Encontro M undial sobre Envelhecimento. M adri; 2002.

11. Pereira M G. Epidemiologia - teoria e prática. Rio de Janeiro: Guanabara Koogan; 2000.

Artigo apresentado em 26/01/2005

Aprovado em 28/06/2006

Versão final apresentada em 09/11/2006 